\title{
Der Raum - einmal systemtheoretisch gesehen
}

\section{Der Raum als Wirklichkeit und der «Raum» als ein Schwarm von Homonymen}

Die Reflexion der Geographen über die Geographie ist seit nahezu zwei Jahrhunderten an "den» Raumbegriff gefesselt. Seitdem es (zumindest in der Geographie) um die «Landschaft» stiller geworden ist, gilt dies noch mehr als zuvor.

Wenn man es aus der historischen Vogelperspektive betrachtet, wie diese Jahrhundertfrage nach «dem» Raum in der geographischen Literatur bisher abgearbeitet wurde, dann sieht man vor allem zwei Lösungsstrategien: Eine naiv-ontologische und eine semantische.

Die ontologisierende Strategie gehört bis heute zur Folklore der Geographen. Folgerichtig erscheint diese Ontologisierung z. B. auch noch einmal in WIRTHS «Theoretischer Geographie». Das erste «Axiom der Geographie» lautet einfach: «Es gibt räumliche Differenzierung» und führt unmittelbar fort: «Der Raum ist...» (WIRTH 1979, S. 282). «Räumliche Differenzierung» bzw. «Raum» ist eine «Realkategorie» und in der «dem Geographen vorgegebenen empirischen Welt» selber «begründet» (vgl. z. B. S. 269). Die Welt ist nun einmal räumlich bzw. räumlich differenziert, und darauf «baut... geographische Forschung auf»(vgl. z. B. S. 282f.). Die Geographie wird an der Struktur der Wirklichkeit festgezurrt.

Die Handikaps dieses Denkstils liegen auf der Hand: Nicht nur objekt- und metasprachliche Ebene, auch Deskription und Präskription werden ununterscheidbar, und vor allem: Dieser Antwortstil erschwert es, ja macht es nahezu unmöglich, den «geographischen Raum» differenzierter, z. B. als eine Mehrzahl von Begriffen oder gar als kontingent zu betrachten, d. h. als einen Begriff, der immer «auch-anders-möglich» und durch (räumliche wie nicht-räumliche) funktionale Äquivalente ersetzbar sein könnte. Nicht zuletzt aus diesem Grund geht von Ontologisierungen oder Reifizierungen dieser Art fast immer eine Blickverengung, nicht selten sogar eine intellektuelle Lähmung und eine Art süßer Hypnose aus.

Demgegenüber ist eine semantische Lösungstrategie weitaus anregender. Als klassischen Text kann man einen vielzitierten Vortrag von Dietrich BARTELS bezeichnen, der 1974 in einem Beiheft der
Geographica Helvetica veröffentlicht worden ist. Dieser Text kann hier nicht referiert werden, nicht einmal überschlägig; es genügt, an den Ausgangsgedanken zu erinnern: Inner- und außerwissenschaftlich, inner- wie außergeographisch gibt es viele Raumbegriffe; die Frage sollte also nicht lauten: «Was ist Raum?», sondern: «Was bedeutet «Raum» in den Sprachen $\mathrm{L}_{1}, \mathrm{~L}_{2}, \ldots, \mathrm{L}_{\mathrm{n}}$ ?» Selbst in der modernen Geographie gibt es mindestens ein halbes Dutzend unterschiedlicher Sprach- und Denkmilieus, in denen «Raum» jeweils etwas sehr Verschiedenes meint. ${ }^{1}$

Eine semantische Analyse dieser Art ist in mehreren Hinsichten nützlich, nicht zuletzt bei der Vorbereitung und Rationalisierung der Entscheidung, auf welchen dieser «Räume» wir uns einlassen wollen, und eine solche Analyse kann sogar nützlich sein, um die eigene Wahl im nachhinein besser zu verstehen. Es handelt sich ja nicht nur um die Wahl eines Wortes: Denn mit der reflektierten und konsequenten Wahl eines Raumbegriffs wählt man auch einen spezifischen Kontext mit - oft sogar ein ganzes Forschungsprogramm oder eine noch weiterreichende Forschungsperspektive. In eben diese Richtung ging auch die Intention, mit der BARTELS selber seine semantischen Differenzierungen vorgenommen hat.

\section{Andere Annäherungen an den «Raum»}

Diese wenigen Andeutungen genügen wohl, um verständlich zu machen, daß und warum der «semantische Denkstil» gegenüber dem «ontologisierenden Denkstil» ungleich mehr Differenzierungs- und Selektionsmöglichkeiten bereitstellt - und wozu das gut ist. Es gibt aber auch noch andere Annäherungen an den «Raum».

Unter diesen zahlreichen «anderen Möglichkeiten» besteht eine der fruchtbarsten darin, die unter Geographen (wenigstens zum Teil) heute doch schon ziemlich verbreitete Auffassung, daß die Sozialgeo-

Gerhard Hard, Prof., Dr., Universität Osnabrück,

Fachbereich Kultur- und Geowissenschaften

Postfach 4469, Neuer Graben/Schloss, D-4500 Osnabrück 
graphie eine Sozialwissenschaft sei, auf eine radikalere Weise ernst zu nehmen. Dann erscheint es nicht mehr so vordringlich, die geographischen Raumbegriffe zu explizieren und zu selektieren, und das gleiche gilt von der blickverengenden, aber noch immer eifrig geübten Tradition, nach spezifisch «räumlichen Systemen» zu suchen (sei es in der Wirklichkeit, sei es in anderen Wissenschaften) und diese dann für die Geographie zu reklamieren. Der Sozial- und Wirtschaftsgeograph, der sich als Sozialwissenschaftler versteht, sollte besser fragen, ob und wo Raumbegriffe und Raumbezüge (1.) in umfassenden sozialwissenschaftlichen Theorien und (2.) in der sozialen Wirklichkeit, in sozialen Systemen auftauchen.

Das ist z. B. die Fragestellung bei KLÜTER (1985) und WERLEN (1986). Beide gingen - unabhängig voneinander - von umfassenden sozialwissenschaftlichen bzw. soziologischen Theoriebildungen aus: WERLEN von handlungstheoretischen Ansätzen, KLÜTER von einerTheorie selbstreferentieller sozialer Systeme, und zwar in der besonderen Form, die sie bei LUHMANN erhalten hat. ${ }^{2}$ Die beiden Autoren kommen auf strukturell verwandten Wegen zu sehr ähnlichen Ergebnissen. Im folgenden beziehe ich mich auf die Arbeit von KLÜTER.

Diese Bezugnahme ist allerdings nicht nur sehr selektiv; um der Kürze und der Prägnanz willen werde ich auch nicht immer säuberlich trennen können, was bloß resümierendes Referat, was verdeutlichende Interpretation und was Entfaltung von Konsequenzen ist. Der Autor mag gelegentlich sogar seine Gedankengänge und Akzentsetzungen (ja vielleicht stellenweise sogar seine Gesamtintention) etwas verschoben finden. Da die Arbeit noch 1986 im Druck vorliegen wird, kann der Leser sich in Bälde selbst ein Bild machen.

\section{Die Inversion der Frage nach der «Raumwirksamkeit»}

Die Pointe und die Pfiffigkeit dieses Zugriffs auf «Räume» und «räumliche Systeme» kann man wohl erst richtig würdigen, wenn man die traditionellen Redeweisen zum Vergleich heranzieht, z. B.: «Als räumliche Systeme können solche Systeme bezeichnet werden, deren Elemente und/oder Relationen im Raum eindeutig fixiert sind, die also einen relativ festen und unveränderlichen Standort haben, und die man dementsprechend lokalisieren, kartieren, abgrenzen kann» (WIRTH 1979, S. 125). Erstens ist diese Definition im ersten Teil des Definiens tautologisch («räumlich» wird durch «Raum» definiert). Zweitens formuliert sie in ihrem zweiten Teil, wo diese Tautologie mit «also» erläutert werden soll, nur eine alltagssprachliche Wortgebrauchsregel (denn wer würde schon etwas «räumlich» nennen, was keinen Standort hat und sich weder lokali- sieren noch begrenzen läßt?). Drittens schließt die Definition eigentümlicherweise schon physische $B e$ wegungen aus (die ja definitionsgemäß keinen «relativ festen und unveränderlichen Standort haben»), und viertens kommt erschwerend und entscheidend hinzu, daß man, wenn schon nicht alles, so doch wenigstens fast alles «lokalisieren, kartieren, abgrenzen» und sogar geometrisieren kann - erstens alle physischen Objekte, aber zweitens auch solche Phänomene, die gar keine räumliche Existenz in diesem Sinne haben, also z. B. soziale Systeme im Sinne von LUHMANN. ${ }^{3}$

Nehmen wir als Beispiel ein sehr einfaches soziales System: Ein Telefongespräch zwischen Erlangen und München, wobei über einen Geographielehrstuhl in München gesprochen wird. Das kann man natürlich auch kartieren oder anderswie geometrisieren, sogar auf vielerleiWeise (z. B. durch zwei bis drei Punkte und ein bis zwei Linien); einem Geographen mögen solche «Kartierungen» von sozialen Systemen sogar naheliegen, jedenfalls fallen sie ihm früh (meist viel zu früh) ein. Dies wäre aber (wie sonst, so auch hier) eine sehr reduktive, um nicht zu sagen: eine stupide Kodierung eines sozialen Systems. Erstens wäre diese räumliche Kodierung unendlich vieldeutig. Zweitens trüge sie so gut wie nichts dazu bei, das betreffende soziale System zu «verstehen" oder zu «erklären», und drittens würde in dieser stupiden Kodierung so gut wie überhaupt nichts Wesentliches über das betreffende soziale System ausgesagt. Das bleibt auch dann so, wenn man raffiniertere räumliche Abbildungen als den «chorischen Raum» wählt - etwa Fotografien. Wie man leicht sieht, steht es ähnlich mit den meisten Verräumlichungen (Kartierungen, Geometrisierungen ...) sozialer Systeme, die von Geographen vorgenommen werden, und mit den «Verräumlichungen» ökologischer Systeme durch geographische Landschaftsökologen ist es nicht besser. Damit «räumliche Information» überhaupt etwas Sinnvolles aussagt, muß man sie durchweg erst einmal sehr reichlich mit "unräumlicher Information» anreichern, und wenn man diese Anreicherung bis zu einem befriedigenden Niveau vorangetrieben hat, ist die «räumliche Information» meistens überflüssig geworden. Der Leser sei aufgefordert, dies anhand des vorgelegten Beispiels zu tun.

Kurz, die zitierte Definition von "räumliche Systeme» schließt so gut wie alles ein, was es so gibt in der Welt. Man kann fast alles, vielleicht sogar alles verräumlichen, sowohl Phänomene der ersten (physischen), der zweiten (mentalen) und der dritten (z. B. der sozialen) Welt - um die Popperschen Termini zu benutzen. Das mag oft ziemlich unfruchtbar sein, aber es geht. Eben deshalb ist die Perspektive «Raum» oder «räumlich» keine sinnvolle Selektion; sie seligiert nicht, zumindest nicht hinreichend. Es nutzt auch nichts, auf bestimmte (erdräumliche, alltägliche, landschaftliche, «menschliche»...) Maß- 
stäbe zu verweisen; der mögliche Inhalt bleibt auch dann ohne Sinn und Grenze.

Wiewohl man also mehr oder weniger alles räumlich projizieren (und insofern als ein «räumliches System» darstellen und betrachten) kann, wird im tatsächlichen sozialen Handeln doch bei weitem nicht (fast) alles, sondern nur einiges räumlich abgebildet: Nur bestimmte Gegenstände (bzw. Gegenstandsklassen) durch bestimmte Instanzen zu bestimmten Zwecken und für bestimmte Adressaten.

Von dieser schlichten Feststellung aus kann man der Frage nach dem Raum einen anderen Sinn geben. Wir suchen nicht mehr naiv-realistisch nach dem «Raum» oder nach den famosen «räumlichen Systemen»; wir fragen vielmehr, welche sozialen Systeme «immer schon» (und längst bevor die Geographen räumeln) welche Raumabstraktionen in welchen Kodes produzieren. Der Blick des Sozialgeographen wird so von der physischen Welt auf die soziale Welt gerichtet - für einen Sozialwissenschaftler doch wohl eine sinnvolle Wendung. Es sei erlaubt, es noch drastischer zu formulieren: Wir glotzen nicht mehr altgeographisch auf die physischeWirklichkeit (oder deren «räumliche Strukturen»), um ausgerechnet dort «das Soziale» (oder die Ökonomie, die Politik oder gar die Kultur) zu finden. Wir richten den Blick vielmehr auf die soziale Welt und fragen, welche räumlichen Kodierungen der Wirklichkeit dort vorgenommen werden, wie diese Raumabstraktionen funktionieren und was sie für welche sozialen Systeme und gesellschaftlichen Praxen leisten.

\section{Wie Räume funktionieren}

KLÜTER illustriert die Funktion von Raumabstraktionen unter anderm am Falle eines Hilfsarbeiters, der in seinen Tätigkeitsbereich eingewiesen werden soll. Das könnte dadurch geschehen, daß ihm Produktionsziel und Produktionsablauf, ja das gesamte Unternehmensprogramm samt zugehöriger Technik erläutert werden. Dann hat er den Durchblick und könnte seine eigene Tätigkeit mit höchstem Verständnis ausführen. Wenn das zu weit geht, könnte man dem Neuling doch wenigstens ein relevantes Unterprogramm erläutern, z. B. die Maschine, die bedient werden soll. Die einfachste Form der Information aber bestünde wohl in einer Darstellung geometrischer Art, einer simplen Planskizze, die z. B. den Grafiken der Zeitgeographen gleichen kann und etwa so zu dekodieren ist: Tu zuerst hier das, bewege dich dann zwei Meter nach rechts und tu dort eine Minute lang jenes, geh dann zurück usw. usf.

Kurz, räumliche Information kann komplexere (technische, soziale, ökonomische ...) Informationen substituieren, und zwar unter Umständen auf eine durchaus effektive Weise. Eben dies ist die Hauptfunktion von "Raum» als ein Element sozialer Kommunikation: Räumliche Kodierung empfiehlt sich vor allem dann, wenn das Funktionieren auch ohne alles «tiefere Verständnis» sichergestellt werden kann und soll, aber trotzdem Selbststeuerung (anstatt direkter Fremdsteuerung) erwünscht ist. Die Geographie selber bietet eine gewisse, nicht allzu entfernte Parallele: Solange sie sich auf räumlich kodierbare («räumliche», «raumwirksame» ... .) Information kapriziert, ist die Information, die sie zu bieten hat, sozial- wie naturwissenschaftlich gesehen entsprechend primitiv; als Schulwissenschaft und Unterrichtsstoff - oder als Instrument der Ausbildung verständnisentlasteter Empfänger administrativer Information - funktionierte sie unter Umständen aber eben deshalb gar nicht so schlecht.

Eine Raumabstraktion bringt also ein Organisationsprogramm auf einen sehr einfachen Kode. Von dieser Raumabstraktion kann man aber keinesfalls auf das ungleich komplexere Programm zurückschließen. Bei minimalen Programmänderungen, ja sogar bei unverändertem Programm können (wenn z. B. eine Maschine bloß ihr Design verändert) ganz neue Raumabstraktionen erforderlich werden, und drastische Programmänderungen können das Funktionieren einer Raumabstraktion (z. B. die «Zeitgeographie» eines Hilfsarbeiters in einer Maschinenhalle) ganz unangetastet lassen. Das ist natürlich nur die Umformulierung der Erfahrung, da $\beta$ «der Raum» - das Räumliche, die Landschaft, die räumlichen oder die landschaftlichen Indikatoren ... - unendlich vieldeutig sind.

$\mathrm{Da} B$ und wie der räumliche Kode (oder eine bestimmte Raumabstraktion) als ein Element sozialer Kommunikation funktioniert, kann man natürlich auch an geographienäheren Beispielen illustrieren, z. B. an einem Bauleitplan. Eine Organisation, hier eine Behörde, will bestimmte, zunächst großenteils anonyme Adressaten (Unternehmer, Bauherren, andere Organisationen) beeinflussen, z. B. im Sinne eines komplexen Stadtentwicklungsprogramms (z. B. Wirtschaftsförderung plus Erhöhung der Wohnattraktivität und vieles mehr). Zu diesem Zweck erstellt die Behörde ein möglichst leicht kommunizierbares, also mit Vorteil ein räumliches Schema, das die Adressaten - die potentiellen Akteure - über ihre Chancen in einem bestimmten Handlungsfeld informieren soll. Potentielle Akteure «analysieren» nun «den Raum», d. h. die vorliegende Raumabstraktion, aber natürlich nicht um des Raumes, sondern um sehr unterschiedlicher, durchweg ganz unräumlicher Ziele willen (der eine will den Betrieb verlagern, der zweite sich selbständig machen, der dritte im Grünen wohnen, der vierte Geld anlegen, der fünfte Reihenhäuser verkaufen ...). Keiner von ihnen will, braucht oder kann die Zwecke (das Programm) des Kartenma- 
chers aus der Raumabstraktion herauslesen; die Kartenleser analysieren den Raum (d. i. die Raumabstraktion) vielmehr, um mehr oder weniger verläßliche Erwartungen über die Folgen eigener Handlungen zu bilden, was wiederum nur gelingt, wenn man - wieder anhand der Karte - die Handlungserwartungen, Handlungen und Handlungsfolgen der erwartbaren Ko-Akteure abzuschätzen vermag. Die Raumabstraktion koordiniert und steuert gerade auch soziales Handeln - d. h. Handeln, das sich am Handeln anderer orientiert.

Die Beispiele illustrieren auch einen weiteren wesentlichen Punkt: «Räumliche Orientierungen anhand von Raumabstraktionen» sind zwar nicht ganz selten in bestimmte Kommunikations- und Handlungssequenzen eingelassen, aber diese Kommunikations- und Handlungssequenzen können keineswegs auf diese Raumabstraktionen und Raumorientierungen zurückgeführt werden (von einigen sozialwissenschaftlich uninteressanten Sonderfällen abgesehen). «Räumliche Orientierungen» und «Raumabstraktionen»- wie weit man diesen $\mathrm{Be}$ griff auch spannen mag - sind so gut wie nie ein taugliches Mittel, um menschliche Aktivitäten (oder gar Handeln) zu erklären oder zu verstehen. ${ }^{4}$

\section{Eine Typologie von Raumabstraktionen}

Wissenschaftler mögen zu der Vorstellung neigen, $\mathrm{da} ß$ Raumkonzepte und Raumgliederungen in irgendwelchen Wissenschaften (z. B. in der Geographie) erfunden und dann «der Gesellschaft» oder «der Praxis» im Bedarfsfalle zur Verwendung übergeben werden. Der normale Weg verläuft indessen andersherum; lange bevor es $z$. B. in der akademischen Geographie landschaftete, zirkulierte diese Raumabstraktion «Landschaft» schon außerhalb der Wissenschaften. Man kann die wissenschaftsgeschichtliche Hypothese wagen, daß bisher alle innergeographischen Raumabstraktionen «von drauBen» kamen und dann innergeographisch - meist sozusagen «in anonymem Auftrag» - weitergepflegt wurden.

Wenn Geographen anfangen zu arbeiten, dann sind also nicht nur die sozialen Systeme, sondern auch deren mehr oder weniger zielführenden Raumabstraktionen immer schon da. Wenn dem so ist, dann liegt es nahe zu fragen, welche Raumabstraktionen zu welchen sozialen Systemen und zu welchen sozialen Kodes gehören. Anders gesagt: Welche Räume sind von ihrem originalen gesellschaftlichen Kontext her in welchem Kode geschrieben und von wem an wen adressiert? Für eine solche Zuordnung übernimmt KLÜTER programmgemäß den LUHMANNschen Theorierahmen; sie kann hier nur andeutungsweise und insoweit illustriert werden, als sie zum Verständnis der Pointe notwendig ist. ${ }^{5}$ LUHMANN unterscheidet (1.) einfache Interaktions- systeme, die wechselseitige Wahrnehmung (sowie mehrstelliges Wahrnehmen dieses Sich-Wahrnehmens) voraussetzen und die nur durch - meist ephemere - Themen strukturiert werden, z. B. «eine Skatrunde, eine Massenversammlung, eine Schlägerei, eine Taxifahrt» (LUHMANN 1975, S. 10); (2.) Organisationen, deren Selektionsprinzip Mitgliedschaft ist und die durch Programme geregelt sind (z. B. Familien, Schulklassen, Unternehmen, Behörden); (3.) Gesellschaften, die bei LuHMANN nicht so sehr (wie in der traditionellen Sozialtheorie) durch «Normen» und «Werte», sondern durch kommunikative Erreichbarkeit definiert und entsprechend nach einschlägigen «symbolisch generalisierten Kommunikationsmedien" differenziert sind: Geld/Eigentum ist z. B. das Kommunikationsmedium (der Kode) des gesellschaftlichen Teilsystems «Wirtschaft», Macht/Recht der Kode des politischen Systems, Wahrheit/Reputation der Kode des Teilsystems Wissenschaft usf. Die Liste dieser Kodes ist offen; Schönheit/Ästhetik, Glaube/Vertrauen, Liebe ... sind z. B. Medien weiterer gesellschaftlicher Teilsysteme (Kunst, Religion, Familie ...).

KLÜTER ordnet nun den Interaktionssystemen die Raumabstraktion "Kulisse», den Organisationen "Programmräume» und den Gesellschaftssystemen «Kommunikations»- bzw. "Sprachräume» zu «Sprache» wieder im weiten Sinne von «Zeichensystem» oder «symbolisch generalisiertem Kommunikationsmedium». Geld, Recht, Kunst, aber auch moderne Naturwissenschaft und Technik (oder noch speziellere Idiome) kann man als solche Sprachen mit heute z. T. weltweiten Sprachräumen auffassen. Im Medium Macht/Recht z. B. werden unterschiedliche Typen von Administrationsräumen gebildet; im Medium Geld/Eigentum entstehen Räume aus Marktpunkten und Erreichbarkeitsinformationen (die KLÜTER «Adressenräume» nennt) sowie Netze, die den Tausch von Waren, Diensten und Kapital abbilden (Liefer- und Vertriebsnetze, die KLÜTER als «Ergänzungsräume» bezeichnet). Weniger eindeutig kann die Raumabstraktion «Grundstück» (eine räumliche Projektion von Eigentums- und Verfügungsrechten) einem bestimmten Medium zugeordnet werden. Alle diese Raumabstraktionen einschließlich der sog. "Grundstücksräume» sind, wie gesagt, simplifizierende Rekodierungen von Texten, deren originale und komplexe Fassung in einer Geld-, Macht- oder Rechtssprache gehalten ist. Es ist auch leicht zu sehen, dass alle diese mehr oder weniger medienspezifischen Raumabstraktionen heute vorwiegend in Form von Programmräumen irgendwelcher (Groß-)Organisationen auftreten.

Außerdem gibt es stärker emotionalisierbare Raumabstraktionen, z. B. in den Kommunikationsmedien des Glaubens/Vertrauens, der Schönheit/ Ästhetik und sogar im Medium der Liebe/Sympathie; auch diese Raumabstraktionen haben sichtlich handlungssteuernde Funktionen, und auch sie 
werden heute weitgehend durch (Groß-)Organisationen, z. B. Administrationen und Bildungssysteme (schon seltener durch soziale Bewegungen) erzeugt und eingesetzt: z. B. die Raumabstraktionen «Vaterland», «Heimat», «Landschaft», «suburbanes Eigenheim-» und «Öko-Idyll».

Gerade die Geographie hat - als Schulfach und als akademische Disziplin - viel zur Verbreitung und Ausgestaltung dieser emotional besetzten (und nicht mehr unbedingt geometrisch abbildbaren) Raumabstraktionen beigetragen, möglicherweise mehr als zur Kenntnis und Wirkung von politisch-juridischen und ökonomischen Räumen. ${ }^{6}$

\section{Die Räume der Geographen}

Wie steht es demgegenüber mit den Raumabstraktionen im Medium «wissenschaftliche Wahrheit» (oder-ersatzweise - im Medium «wissenschaftliche Reputation»)? Für ihre eigenen Räume haben die Geographen ja immer den Anspruch erhoben, da 3 es sich in eben diesem Sinne um die "wissenschaftlichen", die "wahren», zumindest die «objektiven» (intersubjektiv gültigen) Räume handle. Diese innerwissenschaftlich hergestellten Eigenräume der Geographen, die kein institutionalisiertes außerwissenschaftliches Praxiskorrelat besitzen, nennt KLÜTER «(geographische) Idealräume».

Naturräume und Ökotope, aber auch die Eigenräume (Regionen, Netze, Standortoptimierungsmodelle ....) derWirtschafts- und Sozialgeographen werden von ihren Produzenten nicht selten ex-oder implizit als solche «Idealräume» verstanden, die, weil sie aufgrund ihrer «objektiven Herstellungsmethode» «objektiv richtig» seien, eigentlich auch Richtschnur für alle sein müßten, zumindest dann, wenn der Adressat aufgeklärt und dem Gemeinwohl verpflichtet sei. Werden diese Idealräume nicht angenommen, dann sperre sich eben eine schlechte politische Praxis gegen eine gute wissenschaftliche Wahrheit.

Alles bisher Gesagte läuft darauf hinaus, $\mathrm{da} ß$ solche Vorstellungen wenig Sinn haben, und zwar aus vielen Gründen, von denen nur einer genannt sei: Räume sind Instrumente der Handlungsorientierung, die an ganz spezifische Praxen angebunden, aber nur in Sonderfällen über diese hinaus generalisierbar sind. Eine Autokarte taugt nicht für die Flurbereinigung und eine Katasterkarte nicht für eine Autotour, und das gilt analog über die ganze Welt der Raumabstraktionen hin. IhrWert bemißt sich an ihrer Brauchbarkeit und Effektivität relativ zu spezifischen Handlungs- und Organisationsprogrammen sowie relativ zu bestimmten Adressaten, die entweder selber steuern wollen oder (möglichst verständnisentlastet) gesteuert werden sollen. Das binäre Schema "wahr/unwahr» bzw. "wissenschaftlich/unwissenschaftlich» ist hier zweitrangig und i. a. irre- levant. Wissenschaftliche Raum-Kodes, z. B. Kodes für geomorphologische oder landschaftsökologische Karten, sind - bestenfalls - geeignet, die Handlungen kartierender Geographiestudenten zu steuern; außerhalb der Wissenschaft hat sich wohl noch nie ein Mensch, geschweige denn eine produzierende oder verwaltende Organisation ernsthaft an ihnen orientiert (weder ein Kavallerist, noch ein Panzerkommandant; weder ein landwirtschaftlicher oder industrieller Betrieb, noch ein Wasserwirtschaftsamt). Kurz, es gibt keinen allgemeingültigen («wahren») Raumdiskurs, in dem allgemeingültige («wahre») Raumabstraktionen erzeugt werden könnten. Die Frage nach dem Wert und der Relevanz einer Raumabstraktion kann nicht von Wissenschaftlern entschieden, ihre Beantwortung muß vielmehr an die außerwissenschaftlichen Nachfrager delegiert werden.

Auch die Frage nach dem spezifisch "geographischen Raum» bekommt so eine einfache, aber, wie ich glaube, heilsam desillusionierende Antwort, und sie gilt für die ganze, auch für die Physische Geographie. Die praktische Konsequenz besteht vor allem darin, daß vor diesen «geographischen Räumen» zu warnen ist. Wenn man die außergeographisch zirkulierenden Programm- und anderen Räume innergeographisch zu «verwissenschaftlichen» oder anderswie umzudefinieren, also durch Eigen- und Idealräume zu ersetzen versucht, dann gefährdet man fast immer nur den kommunikativen Erfolg der geographischen Produkte. Für dieses - von KLÜTER systematisch konstruierte - Argument gibt es bekanntlich auch gute historische Evidenz. Zwar wimmelt es in der Geographie seit ihrer Akademisierung von objektiven geographischen Raumbegriffen. Die Geschichte lehrt aber, dass sie meistens in der Welt herumzogen wie arbeitslose Schauspieler auf der vergeblichen Suche nach einem Engagement. ${ }^{7}$

\section{Eine Aufgabe für die Sozialgeographie?}

Was wie verräumlicht wird, genauer: welche Raumabstraktionen in der Gesellschaft kursieren, hängt vor allem davon ab, welche Adressaten nach Auffassung welcher Organisationen wie handeln sollen. Wenn diese Organisationen bemerken, da $\beta$ die erwarteten Adressatenhandlungen ausbleiben und deshalb ihr Programm gefährdet ist, wird oft zuerst der Programmraum (der Vordergrund sozusagen) problematisiert und dann erst das Programm. Das ist dann vielleicht der Augenblick, in dem eine Organisation - natürlich nur, falls die organisationsinternen Ressourcen nicht ausreichen - möglicherweise einen Wissenschaftler, wahrscheinlicher aber eine andere Spezialfirma (eine Werbeagentur, ein Planungsbüro, einen Designer oder auch einen Künstler) damit beauftragt, die Programmabstrak- 
tion in eine wirkungsvollere Form zu bringen. «Die Aufgabe des Wissenschaftlers besteht darin, diese Form mit möglichst wenigen und möglichst einfachen Transformationen des von der Organisation (Auftraggeber) vorgegebenen Programms herzustellen» (KLÜTER 1985, S. 160). Raumwissenschaftliche Sozialgeographie könnte nicht viel anderes sein als eine Technik zur Analyse und Rationalisierung von Raumabstraktionen, eine Syntax, Semantik und Pragmatik außerwissenschaftlich brauchbarer räumlicher Kodes, deren Wert aber außerhalb der Wissenschaft vorentschieden und nachgeprüft wird, also nicht im Kode «Wahrheit», sondern z. B. im Kode «Geld» oder «Macht».

Damit ist freilich noch ganz offen, ob das ein eigenständiges Ausbildungs- und Forschungsprogramm hergibt (was KLÜTER anzunehmen scheint); ebenso, welche Auftraggeber man befriedigen und - damit zusammenhängend - wie weit man den Begriff «Raumabstraktion» spannen möchte und könnte. Bunte Karten mit Erläuterungstexten (z. B. über Naturräume oder zentralörtliche Bereiche), mehr oder weniger komplizierte Bildfahrpläne (wie die Zeitgeographen sie produzieren), Gravitationsmodelle, Distanz-Verfalls-Kurven, Diffusions- und Standortoptimierungsmodelle, das sind sicher Raumabstraktionen sogar im engeren Sinne; mindestens ebenso erfolgreich waren Geographen aber oft, wenn sie Landschaften und Heimaten, Vaterlands- und Feindeslandbilder ausmalten, und nicht selten sogar dann, wenn sie das länderkundliche Schema - ebenfalls eine Raumabstraktion im weiteren Sinne - mit allerlei statistischer, grafischer und verbaler Information auffüllten.

\section{Schlußbemerkung}

Soweit mein Versuch, das subtile Argumentationsgeflecht der Dissertation interpretierend zu einem notgedrungen allzu lapidaren Text zu linearisieren. Ich habe versucht, dieser erstaunlichen Erstlingsarbeit auch dadurch die Ehre zu erweisen, daß ich das Provokante und Diskussionswürdige an ihr besonders hervorhob. Von den teils naheliegenden, teils diskussionswürdigen Folgerungen, die der Autor zieht, mußten die meisten ausgespart werden. Kurz, ich empfehle diese Dissertation der Lektüre. Wenn man eine Quintessenz formulieren müßte, dann vielleicht (und unter der Gefahr derTrivialisierung) etwa so: Die Sozialgeographie sollte «Räume» nicht ontologisch als Wirklichkeitsstrukturen oder «empirisch vorfindbare» Wirklichkeiten, auch nicht perzeptionsgeographisch-psychologisch als Wahrnehmungsmuster und Wahrnehmungsbestandteile, sondern sozialwissenschaftlich als $" B e$ standteile sozialer Kommunikation" auffassen, also nicht als Strukturen der physischen Welt oder als
Schemata der Wahrnehmung und des Erlebens, sondern als Schemata der Handlungsorientierung und Handlungskoordination, welche bestimmten sozialen Kodes folgen und immer eng an bestimmte soziale Systeme, vor allem aber an bestimmte Organisationen und deren Programme gebunden sind. Kurz: Der Raum als ein spezifisches soziales Konstrukt, nicht als ein physisches Phänomen (oder eine Struktur der physischen Welt). Als Bestandteil der physischen oder der mentalen Welt kann man «Raum» sicher auch verstehen, aber das dürfte im allgemeinen kein sozialwissenschaftlich fruchtbares Verständnis sein.

\section{Anmerkungen}

1 Diese bedeutungsverschiedenen Raumvokabeln ('Raum bis nRaum) haben zwar z. T. eine gewisse semantische "Familienähnlichkeit»; im Prinzip handelt es sich aber eher um einen Schwarm von Homonymen - d. h., 'Raum und nRaum haben so wenig und so viel miteinander zu tun wie das Schloß eines Fürsten und das Schloß an einer Tür, und es wäre sinnlos, nach einem "gemeinsamen Begriffskern" (oder gar nach einem gemeinsamen Wirklichkeitskern) zu suchen. Auf diese Weise unterschied BARTELS 1974 vier (und 1975 mit einigen zusätzlichen Varianten acht) Raumbegriffe.

2 In welchem Sinn "umfassend" in dem Ausdruck "umfassende Theorie" gemeint ist, definiert LUHMANN kurz und bündig 1984, S. 9.

3 «Soziale Systeme haben . . . keine räumliche Existenz. Sie bestehen aus Kommunikation und aus nichts als Kommunikation", und Kommunikation läßt sich nicht räumlich fixieren (LUHMANN briefl. 12.10.83).

4 Dies gilt - im Gegensatz zu dem, was ich selber schon geschrieben habe - in noch höherem Maße von den "mental maps", denn diese mental maps werden von Perzeptionsund anderen Geographen durchweg nicht einmal als Bestandteile sozialer Kommunikation und sozialen Handelns, sondern als mentale Phänomene konstruiert (und höchstens nachträglich auf soziale Ursachen - Sozialisation usf. - zurückgeführt).

5 Das Parallelisierungsverfahren KLÜTERS wirkt nicht selten ziemlich scholastisch; wir müssen hier aber alle Detailkritik aussparen. Die Termini der Theorie LUHMANNS (die oft vom üblichen sozialwissenschaftlichen Sprachgebrauch abweichen) können im folgenden nicht erläutert, sondern nur angewendet werden.

6 Insofern ist es nur zu verständlich, daß Eugen WIRTH in den letzten Sätzen seiner "Theoretischen Geographie" aus vollem Herzen dazu aufruft, doch ja diese Raumbilder im Medium von Schönheit, Liebe und Glauben nicht zu vergessen, weil nur sie es (wie er mit guten Gründen meint) eigentlich verlohnen, ausgerechnet Geograph zu werden. Auch das, was sich zuweilen "humanistic" und "phenomenological geography" nennt (oder an das Modethema "regionale Identität” hängt), ist vielfach mit Emphase auf dem Weg dorthin zurück, nämlich ins Medium der Schönheit, des Glaubens und der Liebe.

7 Die Naturraum- und Ökotopgliederungen geographischer Landschaftsökologen sind nur einer von vielen deprimierenden Belegen. 


\section{Literatur}

BARTELS, D. (1974): Schwierigkeiten mit dem Raumbegriff. In: Beiheft zu Geographica Helvetica 29, Nr. 2/3.

BARTELS, D. und HARD, G. (1975): Lotsenbuch für das Studium der Geographie als Lehrfach. 2. Aufl., Bonn und Kiel.

KLÜTER, H. (1985): Raum als Element sozialer Kommunikation. Diss. Münster.
LUHMANN, N. (1970, 1975, 1981): Soziologische Aufklärung, Bd. 1, 2 und 3. Opladen.

LUHMANN, N. (1984): Soziale Systeme. Grundriß einer allgemeinen Theorie. Frankfurt a. M.

WIRTH, E. (1979): Theoretische Geographie. Grundzüge einer theoretischen Kulturgeographie. Stuttgart.

WERLEN, B. (1986): Handlungstheoretische Sozialgeographie. Diss. Freiburg/Schweiz. 